\title{
Two-year clinical outcomes after coronary drug- eluting stent placement in Chinese men and women: a multicenter, prospective registry study
}

This article was published in the following Dove Press journal:

Patient Preference and Adherence

5 July 2013

Number of times this article has been viewed

\author{
Rajiv Shrestha' \\ Sandeep Gami' \\ Jing $X u^{2}$ \\ Du-Jiang $X_{i e^{2}}$ \\ Zhi-Zhong Liu \\ Tian Xu' \\ Fei Ye ${ }^{2}$ \\ Shi-Qing Din ${ }^{3}$ \\ Xue-Song Qian ${ }^{4}$ \\ Song Yang ${ }^{5}$ \\ Yue-Qiang Liu ${ }^{6}$ \\ Feng $\mathrm{Li}^{7}$ \\ Ai-Ping Zhang ${ }^{8}$ \\ Shao-Liang Chen ${ }^{2}$ \\ 'Nanjing Medical University, \\ Nanjing, ${ }^{2}$ Nanjing First Hospital, \\ Nanjing Medical University, Nanjing, \\ ${ }^{3}$ Huainan Xinhua Hospital, Huainan, \\ ${ }^{4} Z$ hangjiagang People's Hospital, \\ Zhangjiagang, ${ }^{5}$ Yixin People's Hospital, \\ Yixin, JJintan People's Hospital, Jintan, \\ ${ }^{7}$ Huainan Oriental General Hospital, \\ Huainan, ${ }^{8}$ Huainan People's Hospital, \\ Huainan, People's Republic of China
}

Correspondence: Shao-Liang Chen Nanjing First Hospital, Nanjing Medical

University, Nanjing 210006, People's

Republic of China

Tel +862552208048

Fax +86 25 52208048

Email chmengx@I26.com
Background: Previous studies have reported a discrepancy in baseline characteristics and outcomes after percutaneous coronary intervention between men and women. However, this finding has never been verified in the Chinese population. The present study analyzed twoyear clinical outcomes after placement of coronary drug-eluting stents in Chinese men and women.

Methods: From January 2005 to December 2010, a total of 3804 Chinese patients (2776 men, 1028 women) who underwent drug-eluting stent implantation were studied prospectively. The primary endpoint was the composite major adverse cardiac event (MACE) rate, including myocardial infarction, cardiac death, and target vessel revascularization at two years. Stent thrombosis served as the safety endpoint. Propensity score matching was used to compare the adjusted MACE rate between the two groups.

Results: At two-year follow-up, unadjusted rates of myocardial infarction, non-ST segment elevation myocardial infarction, target vessel revascularization, and MACE were significantly different between men $(6.84 \%, 4.6 \%, 13.1 \%$, and $21.7 \%$, respectively) and women $(3.8 \%[P=0.001], 2.0 \%[P<0.001] 10.3 \%[P=0.025]$, and $16.3 \%$ $[P<0.001]$, respectively). After propensity score matching, there were no significant differences in composite MACE and individual endpoints at two years between the genders.

Conclusion: Despite all the unfavorable risk factor clustering in women and complex coronary disease in men, the two-year clinical outcomes after coronary stent placement were comparable between Chinese women and men.

Keywords: drug-eluting stent, major adverse cardiac event, gender difference, clinical follow-up

\section{Introduction}

In the modern era, percutaneous coronary intervention (PCI) with a drug-eluting stent has been used extensively for treatment of coronary artery disease, with dramatically reduced restenosis and target lesion revascularization rates $^{1,2}$ when compared with the bare metal stent. Previous studies ${ }^{3}$ have reported a gender-based difference in major adverse cardiac events (MACE) after index PCI procedures. However, comparative data concerning differences in clinical outcomes after drug-eluting stent implantation according to gender are still not available for the Chinese population. Therefore, the present study aimed to analyze the impact of gender on clinical outcomes after drug-eluting stent implantation in Chinese men and women. 


\section{Materials and methods \\ Patient population}

Patients with acute myocardial infarction, unstable angina, stable angina, or silent ischemia who underwent PCI at our centers between January 2005 and December 2010 were prospectively registered. Patients with bare metal stent implantation were excluded from the analysis. In total, 3804 patients (2776 men, 1028 women) were recruited for this study.

\section{$\mathrm{PCl}$ procedures}

Coronary angiography and PCI with stent implantation were performed via the transfemoral or transradial approach. The decision to use intravascular ultrasound and selection of a sirolimus-eluting or paclitaxel-eluting stent was made by experienced interventional cardiologists. Angiographic success was defined as a residual stenosis $\leq 30 \%$ by visual estimation in the presence of grade 3 TIMI (Thrombolysis in Myocardial Infarction) flow. Procedural success was defined as achievement of angiographic success in the absence of inhospital events.

\section{Definitions}

The primary endpoint was a composite of MACE, including myocardial infarction, cardiac death, and target vessel revascularization, at two years. Stent thrombosis served as the safety endpoint. Diagnosis of myocardial infarction was based on American College of Cardiology/American Heart Association guidelines. ${ }^{4,5}$ Any unexplained death was diagnosed as being cardiac unless a noncardiac cause was identified. Target lesion revascularization was defined as repeat intervention on the stented segment, including the $5 \mathrm{~mm}$ proximal or distal to the stent. Target vessel revascularization was defined as repeat revascularization of the same vessel treated in the index procedure. Stent thrombosis was defined according to the Academic Research Consortium definition. ${ }^{6}$ Definite stent thrombosis was defined as angiographic or pathologic confirmation of partial or total thrombotic occlusion within the peristent region and at least one of the following additional criteria: symptoms of acute ischemia; ischemic changes on electrocardiography; and elevated cardiac enzymes. Probable stent thrombosis was defined as any unexplained death within 30 days of stent implantation, or any myocardial infarction related to documented acute ischemia in the territory of the implanted stent without angiographic confirmation of stent thrombosis and in the absence of any other obvious cause.

\section{Follow-up}

Patients were routinely followed up at one month, one year, and two years. Patients were followed up on an outpatient basis, by telephone contact, or via their relatives. The records for patients who visited other medical centers were also obtained. All clinical and imaging data were recorded in our database. Patients were advised to undergo repeat angiography once during the year following the procedure, but this was not done routinely for all patients. Earlier angiographic surveillance was performed if clinically indicated. Clinically driven target lesion or vessel revascularization was performed if there was at least $50 \%$ instent restenosis coupled with the presence of typical angina or exertional dyspnea of new onset.

\section{Medications}

All patients were pretreated with $300 \mathrm{mg}$ of both aspirin and clopidogrel. Patients were maintained on aspirin $100 \mathrm{mg}$ and clopidogrel $100 \mathrm{mg}$ once daily. All patients were recommended to take aspirin $100 \mathrm{mg}$ daily lifelong. Clopidogrel $75 \mathrm{mg}$ per day was prescribed for at least 12 months, according to the clinical guidelines at that time.

\section{Statistical analysis}

The Kolmogorov-Smirnov test was used to assess the distribution of continuous variables. Continuous variables were expressed as the mean \pm standard deviation or median, and were compared using the Student's $t$-test (for normal data) and the Mann-Whitney $U$ test (for non-normally distributed variables) as appropriate. Categorical variables are presented as frequencies or percentages, which were compared by chi-square or Fisher's exact test. Survival curves were generated using the Kaplan-Meier method, and were compared using the log-rank test. A propensity score analysis was performed to minimize any selection bias arising from differences in baseline characteristics according to gender. Variables included in the logistic regression model to calculate the propensity score were age, gender, body mass index, hypertension, diabetes, hyperlipidemia, acute myocardial infarction, chronic renal insufficiency, left ventricular ejection fraction, multivessel disease, left main stem coronary artery disease, thrombus-containing lesion, chronic total occlusion, transradial approach, incomplete revascularization, type of stent, post-stenting TIMI grade, number of stents, stent diameter, stent length, procedural time, and contrast volume. The $\mathrm{C}$ statistic was 0.67 and the Hosmer-Lemeshow $P$ value was 0.114 , confirming good discrimination and calibration of the propensity score model. The new propensity score was then incorporated into Cox 
proportional hazards regression models as a covariate to assess the clinic outcome for women versus men. In addition, to reduce the effect of treatment selection bias and potential confounding in this observational study, we performed rigorous adjustment for significant differences in the baseline characteristics of patients, with propensity score matching using the following algorithm: a 1:1 optimal match with $\mathrm{a} \pm 0.03$ caliper and no replacement. Clinical outcomes in the matched population were analyzed with Cox proportional hazards regression stratified on matched pairs. Multivariable Cox proportional hazards regression modeling was performed to identify independent predictors of the primary endpoint (MACE, including cardiac death, myocardial infarction, target lesion or vessel revascularization) with purposeful selection of covariates. Variables associated at univariate analysis (all $P$ values $\leq 0.1$ ) and those judged to be of clinical importance from previous published reports were eligible for inclusion in the multivariable model building process. The goodness of fit of the Cox multivariable model was assessed using the Grønnesby-Borgan-May test. Results are reported as the hazards ratio with the $95 \%$ confidence interval and $P$ value. All statistical analysis was performed using Stata version 12.0 software (Stata Corporation, College Station, TX, USA).

\section{Results}

\section{Baseline clinical characteristics}

Of the 3804 patients, 2776 were men and 1028 were women. Baseline characteristics are shown in Table 1. Women were characterized by older age, lower height/weight, higher blood pressure, fasting blood glucose, and interleukin-6 levels, more frequent dyslipidemia, renal dysfunction, use of calcium channel blockers, and angiotensin receptor blockers, and well preserved left ventricular function. In contrast, male patients were found to have a significantly greater incidence of ST elevation myocardial infarction (STEMI) compared with women $(P<0.001)$.

\section{Angiographic and procedural features}

The angiographic and procedural features of the patients are shown in Table 2. Men appeared to have more frequent complex lesions. Left main stem lesions $(12.5 \%$ in males versus $9.7 \%$ in females, $P=0.017)$, bifurcations $(34.1 \%$ versus $30 \%, P=0.016$ ), and thrombus-containing lesions (13.1\% versus $6.1 \%, P \leq 0.001)$, resulting in more stents being needed $(1.99 \pm 1.08$ versus $1.85 \pm 1.01, P<0.001)$ and a longer procedural time $(49.48 \pm 28.45$ versus $45.67 \pm 24.42$, $P<0.001)$, higher contrast volume $(211.17 \pm 78.80$ versus
Table I Baseline clinical characteristics

\begin{tabular}{|c|c|c|c|}
\hline & $\begin{array}{l}\text { Women } \\
(n=1028)\end{array}$ & $\begin{array}{l}\text { Men } \\
(n=2776)\end{array}$ & $P$ value \\
\hline Age, years & $66.17 \pm 9.10$ & $63.76 \pm 10.56$ & $<0.001$ \\
\hline Height (cm) & $159.14 \pm 5.10$ & $169.31 \pm 4.97$ & $<0.001$ \\
\hline Weight (kg) & $62.30 \pm 9.22$ & $70.75 \pm 9.75$ & $<0.001$ \\
\hline Body mass index, $\mathrm{kg} / \mathrm{m}^{2}$ & $24.58 \pm 3.41$ & $24.64 \pm 2.96$ & 0.634 \\
\hline Hypertension, n (\%) & 787 (76.6) & $1903(68.6)$ & $<0.001$ \\
\hline Systolic BP & $136.60 \pm 20.14$ & $133.76 \pm 20.23$ & $<0.001$ \\
\hline Diastolic BP & $79.66 \pm 11.15$ & $80.67 \pm|2.3|$ & 0.017 \\
\hline Hyperlipidemia, n (\%) & $4 I I(40.0)$ & 921 (33.2) & $<0.001$ \\
\hline $\begin{array}{l}\text { Fasting total } \\
\text { cholesterol, } \mathrm{mmol} / \mathrm{L}\end{array}$ & $4.57 \pm 0.87$ & $4.30 \pm 0.83$ & $<0.001$ \\
\hline Fasting LDL, mmol/L & $2.94 \pm 0.78$ & $2.75 \pm 0.75$ & $<0.001$ \\
\hline Fasting HDL, mmol/L & $1.0 \pm 0.08$ & $0.99 \pm 0.08$ & $<0.001$ \\
\hline $\begin{array}{l}\text { Fasting triglycerides, } \\
\mathrm{mmol} / \mathrm{L}\end{array}$ & $1.56 \pm 0.24$ & $1.57 \pm 0.37$ & 0.461 \\
\hline Diabetes, n (\%) & $356(34.6)$ & $796(28.7)$ & $<0.001$ \\
\hline Fasting glucose, $\mathrm{mmol} / \mathrm{L}$ & $6.78 \pm 2.06$ & $6.72 \pm 2.06$ & 0.416 \\
\hline Insulin & $96(27.0)$ & $196(24.6)$ & 0.420 \\
\hline Oral antiglycemics & $248(69.7)$ & $533(67.0)$ & 0.376 \\
\hline $\begin{array}{l}\text { Chronic renal } \\
\text { insufficiency, } \mathrm{n}(\%)\end{array}$ & $129(12.5)$ & $266(9.6)$ & 0.008 \\
\hline $\begin{array}{l}\text { Estimated GFR, } \\
\mathrm{mL} / \mathrm{min} / \mathrm{l} .73 \mathrm{~m}^{2}\end{array}$ & $67.73 \pm 14.95$ & $69.96 \pm 20.46$ & 0.001 \\
\hline Presentation, n (\%) & & & $<0.001$ \\
\hline Stable angina & $168(16.3)$ & $365(13.1)$ & 0.013 \\
\hline Unstable angina & $66 I(64.3)$ & $1523(54.9)$ & $<0.001$ \\
\hline STEMI & $140(\mid 3.6)$ & $685(24.7)$ & $<0.001$ \\
\hline Non-STEMI & $59(5.7)$ & $203(7.3)$ & 0.097 \\
\hline $\begin{array}{l}\text { Congestive heart failure, } \\
\text { n (\%) }\end{array}$ & $223(21.7)$ & $562(20.2)$ & 0.343 \\
\hline $\begin{array}{l}\text { Left ventricular ejection } \\
\text { fraction, } \%\end{array}$ & $59.34 \pm 7.84$ & $57.91 \pm 8.42$ & $<0.001$ \\
\hline Interleukin 6 & $9.83 \pm 1.77$ & $9.74 \pm 1.96$ & 0.030 \\
\hline $\begin{array}{l}\text { Highly sensitive C-reactive } \\
\text { protein }\end{array}$ & $26.62 \pm 4.67$ & $27.46 \pm 4.57$ & 0.550 \\
\hline \multicolumn{4}{|l|}{ Medication, n (\%) } \\
\hline Statins & $138(13.4)$ & $374(13.5)$ & 1.0 \\
\hline Beta-blockers & $173(16.8)$ & $397(14.3)$ & 0.058 \\
\hline Diuretics & $68(6.6)$ & $146(5.3)$ & 0.113 \\
\hline Calcium channel blockers & $340(33.1)$ & $767(27.6)$ & 0.001 \\
\hline $\begin{array}{l}\text { Angiotensin-converting } \\
\text { enzyme blockers }\end{array}$ & $|3|(\mid 2.7)$ & $399(14.4)$ & 0.206 \\
\hline $\begin{array}{l}\text { Angiotensin receptor } \\
\text { blockers }\end{array}$ & $107(10.4)$ & $228(8.2)$ & 0.039 \\
\hline
\end{tabular}

Abbreviations: BP, blood pressure; GFR, glomerular filtration rate; STEMI, STsegment elevation myocardial infarction; LDL, low-density lipoprotein cholesterol; $\mathrm{HDL}$, high-density lipoprotein cholesterol.

$201.98 \pm 57.98, P<0.001)$, and less complete revascularization (59.7\% versus $66.3 \%, P \leq 0.001)$. There was also a difference in the percentage of transradial approaches used between men and women ( $60.7 \%$ versus $55.9 \%$, respectively, $P=0.008)$, with a difference in stent diameter $(3.13 \pm 0.44$ versus $3.08 \pm 045, P=0.003)$ and stent length $(52.36 \pm 31.72$ versus $47.40 \pm 30.68, P<0.001)$ between the genders. 
Table 2 Angiographic and procedural features

\begin{tabular}{|c|c|c|c|}
\hline & $\begin{array}{l}\text { Women } \\
(n=1028)\end{array}$ & $\begin{array}{l}\text { Men } \\
(n=2776)\end{array}$ & $P$ value \\
\hline Diseased vessels (n) & $1.30 \pm 0.56$ & $1.33 \pm 0.57$ & 0.223 \\
\hline One & $770(74.9)$ & $20 I I(72.4)$ & 0.138 \\
\hline Two & $199(19.4)$ & $601(21.6)$ & 0.128 \\
\hline Three & $59(5.7)$ & $164(5.9)$ & 0.877 \\
\hline \multicolumn{4}{|l|}{ Distribution of lesions (n, \%) } \\
\hline LAD & $684(66.5)$ & $1788(64.4)$ & 0.235 \\
\hline LCX & $247(24.0)$ & $668(24.1)$ & 1.0 \\
\hline RCA & $309(30.1)$ & $895(32.2)$ & 0.209 \\
\hline LM & $100(9.7)$ & $348(12.5)$ & 0.017 \\
\hline CTO lesion & $5(0.5)$ & $12(0.4)$ & 0.788 \\
\hline Distribution & & & 0.520 \\
\hline LAD & $4(0.3)$ & $10(0.3)$ & \\
\hline LCX & $\mathrm{I}(0.1)$ & I (0.03) & \\
\hline $\mathrm{RCA}$ & $2(0.2)$ & $3(0.1)$ & \\
\hline$>I$ CTO & I $(0.1)$ & $2(0.07)$ & 1.0 \\
\hline Bifurcating lesions ( $\mathrm{n}, \%)$ & $308(30.0)$ & $947(34.1)$ & 0.016 \\
\hline Thrombus-containing & $63(6.1)$ & $364(13.1)$ & $<0.001$ \\
\hline Transradial approach $(\mathrm{n}, \%)$ & 575 (55.9) & $1685(60.7)$ & 0.008 \\
\hline Sirolimus-eluting stent & $879(85.5)$ & $2388(86.0)$ & 0.714 \\
\hline $\begin{array}{l}\text { Complete revascularization } \\
(\mathrm{n}, \%)\end{array}$ & $682(66.3)$ & $1656(59.7)$ & $<0.001$ \\
\hline $\begin{array}{l}\text { Post-stenting TIMI grade } \\
(\mathrm{n}, \%)\end{array}$ & & & 0.655 \\
\hline 0 & II (I.I) & $37(1.3)$ & \\
\hline I & 0 & $4(0.1)$ & \\
\hline 2 & $8(0.8)$ & $17(0.6)$ & \\
\hline 3 & $1009(98.2)$ & $2718(97.9)$ & \\
\hline Stents (n) & $1.85 \pm 1.01$ & $1.99 \pm 1.08$ & $<0.001$ \\
\hline BP-SES (n, \%) & $350(34.0)$ & $1037(37.4)$ & 0.060 \\
\hline Stent diameter $(\mathrm{mm})$ & $3.08 \pm 0.45$ & $3.13 \pm 0.44$ & 0.003 \\
\hline Stent length (mm) & $47.40 \pm 30.68$ & $52.36 \pm 31.72$ & $<0.001$ \\
\hline Post-dilation & $948(92.2)$ & $2540(91.5)$ & 0.475 \\
\hline Procedural time (minutes) & $45.67 \pm 24.42$ & $49.48 \pm 28.45$ & $<0.001$ \\
\hline Contrast volume $(\mathrm{mL})$ & $201.98 \pm 57.98$ & $211.17 \pm 78.80$ & $<0.001$ \\
\hline
\end{tabular}

\section{Cumulative MACE during one year of follow-up}

Nearly $94.7 \%$ of patients underwent repeat angiography during one year of follow-up (Table 3). The rate of inhospital myocardial infarction was higher in men at $6.3 \%$, mainly because of an increment in the rate of non-STEMI (4.5\%), compared with women $(3.3 \%$ and $1.9 \%$, respectively, both $P<0.001)$. The composite inhospital rate for MACE was significantly different between women and men $(5.2 \%$ versus $7.5 \%, P=0.011)$.

\section{Cumulative MACE during two years of follow-up}

At two years after the index PCI procedure (Table 4), unadjusted rates of myocardial infarction, non-STEMI,
Table 3 Cumulative major adverse cardiac events during one year of follow-up

\begin{tabular}{|c|c|c|c|}
\hline & $\begin{array}{l}\text { Women } \\
(n=1028)\end{array}$ & $\begin{array}{l}\text { Men } \\
(n=2776)\end{array}$ & $P$ value \\
\hline Repeat angiography (n, \%) & $973(94.6)$ & 2634 (94.9) & 0.805 \\
\hline Days from $\mathrm{PCl}$ to angiography & $244(8 I)$ & $244(69)$ & 0.154 \\
\hline \multicolumn{4}{|l|}{ Inhospital, n (\%) } \\
\hline MI & $34(3.3)$ & $175(6.3)$ & $<0.001$ \\
\hline STEMI & $14(1.4)$ & $51(1.8)$ & 0.330 \\
\hline Non-STEMI & $20(1.9)$ & $124(4.5)$ & $<0.001$ \\
\hline Death & $22(2.1)$ & $40(1.4)$ & 0.149 \\
\hline Cardiac & $20(1.9)$ & $38(1.4)$ & 0.232 \\
\hline TLR & $5(0.5)$ & $16(0.6)$ & 0.812 \\
\hline CABG & $3(0.3)$ & $7(0.3)$ & 0.735 \\
\hline TVR & $9(0.9)$ & $25(0.9)$ & 1.0 \\
\hline MACE & $53(5.2)$ & $208(7.5)$ & 0.011 \\
\hline Stent thrombosis & $4(0.4)$ & $18(0.6)$ & 0.472 \\
\hline Definite & $3(0.3)$ & $14(0.5)$ & 0.584 \\
\hline Probable & $\mathrm{I}(0.1)$ & $4(0.1)$ & 1.0 \\
\hline \multicolumn{4}{|l|}{ At one month, $\mathrm{n}(\%)$} \\
\hline MI & $33(3.2)$ & $175(6.3)$ & $<0.001$ \\
\hline STEMI & $13(1.3)$ & $51(1.8)$ & 0.257 \\
\hline Non-STEMI & $20(1.9)$ & $124(4.5)$ & $<0.001$ \\
\hline Death & $28(2.7)$ & $66(2.4)$ & 0.557 \\
\hline Cardiac & $23(2.2)$ & $61(2.2)$ & 1.0 \\
\hline TLR & $5(0.5)$ & $18(0.6)$ & 0.646 \\
\hline CABG & $3(0.3)$ & $8(0.3)$ & 1.0 \\
\hline TVR & $9(0.9)$ & $28(1.0)$ & 0.853 \\
\hline MACE & $55(5.4)$ & $223(8.0)$ & 0.005 \\
\hline Stent thrombosis & $7(0.7)$ & $24(0.9)$ & 0.687 \\
\hline Definite & $3(0.3)$ & $15(0.5)$ & 0.430 \\
\hline Probable & $4(0.4)$ & $9(0.3)$ & 0.758 \\
\hline \multicolumn{4}{|l|}{ At one year, $\mathrm{n}(\%)$} \\
\hline MI & $39(3.8)$ & $184(6.6)$ & 0.001 \\
\hline STEMI & $18(1.8)$ & $57(2.1)$ & 0.602 \\
\hline Non-STEMI & $21(2.0)$ & $127(4.6)$ & $<0.001$ \\
\hline Death & $55(5.4)$ & $|4|(5.1)$ & 0.741 \\
\hline Cardiac & $38(3.7)$ & $99(3.6)$ & 0.922 \\
\hline TLR & $72(7.0)$ & $230(8.3)$ & 0.200 \\
\hline CABG & $6(0.6)$ & $17(0.6)$ & 1.0 \\
\hline TVR & $94(9.1)$ & $315(11.3)$ & 0.052 \\
\hline MACE & $152(14.8)$ & $550(19.8)$ & $<0.001$ \\
\hline Stent thrombosis & $8(0.8)$ & $32(1.2)$ & 0.374 \\
\hline Definite & $3(0.3)$ & $15(0.5)$ & 0.430 \\
\hline Probable & $4(0.4)$ & $9(0.3)$ & 0.758 \\
\hline Possible & $\mathrm{I}(0.1)$ & $8(0.3)$ & 0.459 \\
\hline Late & $2(0.2)$ & $8(0.3)$ & 1.0 \\
\hline
\end{tabular}

Abbreviations: $\mathrm{CABG}$, coronary artery bypass graft; MACE, major adverse cardiac events; STEMI, ST-segment elevation myocardial infarction; TLR, target lesion revascularization; TVR, target vessel revascularization; MI, myocardial infarction; $\mathrm{PCl}$ percutaneous coronary intervention.

target vessel revascularization, and MACE were significantly different between men $(6.84 \%, 4.6 \%, 13.1 \%$, and $21.7 \%)$ and women $(3.8 \%[P=0.001], 2.0 \%[P<0.001] 10.3 \%$ $[P=0.025]$, and $16.3 \%[P<0.001]$, respectively). Other clinical outcomes, including death, target lesion revascularization, coronary artery bypass grafting, and stent 
Table 4 Cumulative major adverse cardiac events during two years of follow-up

\begin{tabular}{|c|c|c|c|c|}
\hline & $\begin{array}{l}\text { Women } \\
(n=1028)\end{array}$ & $\begin{array}{l}\text { Men } \\
(n=2776)\end{array}$ & Hazards ratio & $P$ value \\
\hline \multicolumn{5}{|l|}{ At two years, $n(\%)$} \\
\hline MI & $39(3.8)$ & $190(6.84)$ & $0.55(0.39-0.78)$ & 0.001 \\
\hline STEMI & $18(1.8)$ & $61(2.2)$ & $0.79(0.47-1.33)$ & 0.371 \\
\hline Non-STEMI & $21(2.0)$ & $129(4.6)$ & $0.44(0.28-0.69)$ & $<0.00$ I \\
\hline Death & $67(6.5)$ & $179(6.4)$ & I.0I (0.77-I.34) & 0.927 \\
\hline Cardiac & $42(4.1)$ & $109(3.9)$ & I.04 (0.73-I.49) & 0.816 \\
\hline TLR & $82(8.0)$ & $266(9.6)$ & $0.83(0.65-1.06)$ & 0.139 \\
\hline CABG & $6(0.6)$ & $19(0.7)$ & $0.85(0.34-2.14)$ & 0.737 \\
\hline TVR & $106(10.3)$ & $363(13.1)$ & $0.78(0.63-0.97)$ & 0.025 \\
\hline MACE & $168(16.3)$ & $604(21.7)$ & $0.73(0.6 \mathrm{I}-0.86)$ & $<0.001$ \\
\hline Stent thrombosis & $9(0.9)$ & $33(1.2)$ & $0.74(0.35-1.54)$ & 0.415 \\
\hline Definite & $3(0.3)$ & $15(0.5)$ & $0.54(0.16-1.87)$ & 0.330 \\
\hline Probable & $4(0.4)$ & $2(0.4)$ & $1.20(0.37-3.90)$ & 0.761 \\
\hline Possible & $2(0.2)$ & $9(0.3)$ & $0.60(0.13-2.77)$ & 0.511 \\
\hline Late or very late & $3(0.3)$ & $9(0.3)$ & $0.90(0.24-3.31)$ & 0.871 \\
\hline \multicolumn{5}{|c|}{ After propensity score matching* } \\
\hline Ml & $24(6.6)$ & $22(6.0)$ & I.I (0.60-2.02) & 0.758 \\
\hline STEMI & $9(2.5)$ & $8(2.2)$ & $1.13(0.43-2.92)$ & 0.808 \\
\hline Non-STEMI & I5 (4.I) & 14 (3.8) & $\mathrm{I} .08(0.5 \mathrm{I}-2.29)$ & 0.847 \\
\hline Death & $23(6.3)$ & $24(6.6)$ & $0.95(0.52-1.76)$ & 0.876 \\
\hline Cardiac & $16(4.4)$ & I7 (4.7) & $0.93(0.45-1.93)$ & 0.853 \\
\hline TLR & $24(6.6)$ & $30(8.2)$ & $0.79(0.45-1.37)$ & 0.397 \\
\hline CABG & 0 & $2(0.6)$ & & 0.499 \\
\hline TVR & $33(9.0)$ & 43 (II.8) & $0.74(0.46-1.20)$ & 0.227 \\
\hline MACE & $61(16.7)$ & $75(20.6)$ & $0.77(0.53-1.12)$ & 0.183 \\
\hline Stent thrombosis & $5(1.4)$ & $3(0.8)$ & $1.67(0.40-6.97)$ & 0.484 \\
\hline Definite & $2(0.6)$ & I $(0.3)$ & $0.20(0.18-22.6)$ & 0.571 \\
\hline Probable & $2(0.6)$ & $2(0.6)$ & $1.0(0.14-7.10)$ & 1.0 \\
\hline Late or very late & I $(0.3)$ & 0 & & 1.0 \\
\hline
\end{tabular}

Note: $*_{n}=365$ in both women and men.

Abbreviations: CABG, coronary artery bypass graft; MACE, major adverse cardiac events; STEMI, ST-segment elevation myocardial infarction; TLR, target lesion revascularization; TVR, target vessel revascularization; MI, myocardial infarction.

thrombosis (including definite, probable, possible, late, or very late), were similar between men and women.

After propensity score matching, 365 pairs of men and women were identified (Table 5). There were no significant differences in individual endpoints (myocardial infarction, STEMI, non-STEMI, death, target lesion or vessel revascularization, coronary artery bypass grafting) or composite MACE between men and women. Stent thrombosis was $1.4 \%$ in women versus $0.8 \%$ in men

Table 5 Predictors of primary endpoints, ie, major adverse cardiac events, at Cox multivariate analysis

\begin{tabular}{llll}
\hline Predictors & HR & $\mathbf{9 5 \%} \mathbf{C l}$ & P value \\
\hline Women versus men & 0.76 & $0.58-1.0 \mathrm{I}$ & 0.058 \\
Acute myocardial infarction & $\mathrm{I} .63$ & $\mathrm{I} .2 \mathrm{I}-2.18$ & $0.00 \mathrm{I}$ \\
Transradial approach & 0.74 & $0.58-0.95$ & $0.00 \mathrm{I}$ \\
Sirolimus-eluting stent & 0.62 & $0.42-0.9 \mathrm{I}$ & 0.015 \\
Complete revascularization & 0.77 & $0.60-0.99$ & 0.042 \\
Stent diameter & 0.53 & $0.37-0.7 \mathrm{I}$ & $<0.00 \mathrm{I}$ \\
\hline
\end{tabular}

Abbreviations: $\mathrm{Cl}$, confidence interval; $\mathrm{HR}$, hazards ratio.
$(P=0.484)$. Cox multivariable analysis demonstrated that myocardial infarction (hazards ratio 1.63, 95\% confidence interval $1.21-2.18, P=0.001$ ) was the only independent risk factor for the composite of MACE (Figure 1).

\section{Discussion}

The present study yielded several major findings. First, there was an obvious discrepancy in baseline clinical and lesional characteristics between Chinese women and men, and second, male patients had higher unadjusted rates of myocardial infarction, non-STEMI, target vessel revascularization, and MACE. However, this difference between men and women became narrow after propensity matching score.

Previous studies have shown that women have more risk factors and are older than men at the time of presentation with acute coronary syndrome. ${ }^{7,8}$ This discrepancy is consistent with the delayed onset of coronary artery disease in women, in whom estrogen is thought to have a cardioprotective effect. ${ }^{9}$ In the current Chinese analysis, 


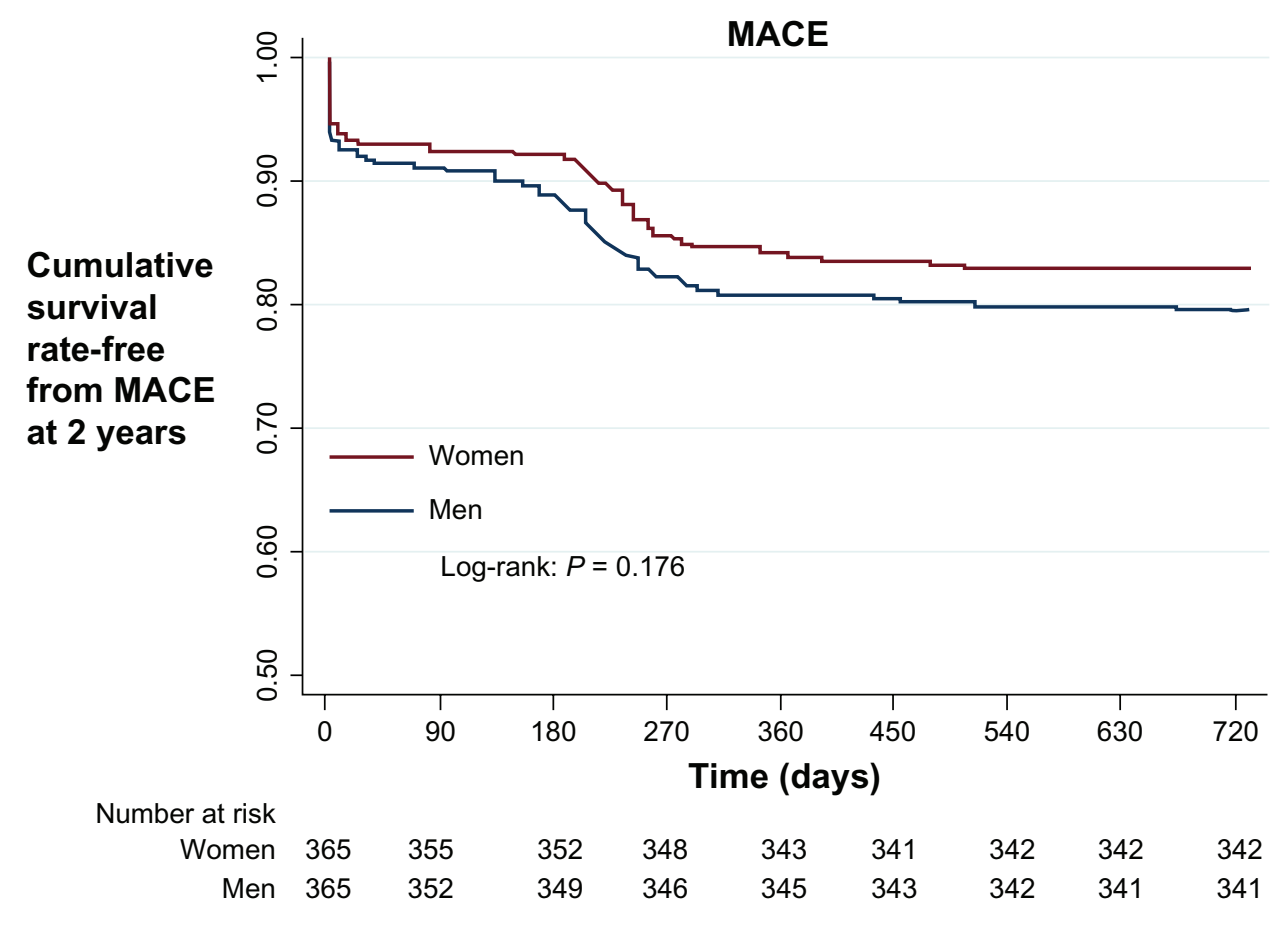

Figure I Kaplan-Meier survival curve for 2-year follow up. Abbreviation: MACE, major adverse cardiac events.

female patients had similar risk factors and were older than male patients. Several previous studies ${ }^{10-14}$ have reported poor outcomes and a higher incidence of MACE after PCI in women with diabetes mellitus, although a study by Ogita et $\mathrm{al}^{15}$ showed no significance difference between the genders after elective drug-eluting stent implantation in diabetic patients. As in our study, diabetes mellitus did not emerge as an independent predictor of MACE in women after implantation of a drug-eluting stent. This can be explained by the fact that previous studies did not report the type of stent used and did not perform propensity score matching. The SYNERGY (Superior Yield of the New strategy of Enoxaparin, Revascularization and GlYcoprotein IIb/IIIa Inhibitors) trial ${ }^{16}$ concluded that chronic kidney disease (derived from the estimated glomerular filtration rate) was predictive of 30-day mortality, myocardial infarction, and bleeding, which is in contrast with our finding that, although women had a higher rate of renal insufficiency compared with men, the unadjusted myocardial infarction rate was higher in men than in women. This is in line with the results of a study by Kralev et al, ${ }^{17}$ who reported that female gender did not emerge as an independent predictor of MACE despite the worse baseline clinical features seen in women. Several studies $^{18,19}$ have indicated no difference in clinical outcome between men and women after invasive treatment. Further, Schuhlen et $\mathrm{al}^{20}$ found that female gender conferred no excess risk for MACE after PCI. Lansky et $\mathrm{al}^{21}$ concluded that despite having more comorbid risk factors than men, women were likely to have less extensive coronary artery disease by both angiographic and intravascular ultrasound measures; however, they found no significant difference in MACE at three years between men and women. Consistent with a previous study ${ }^{21}$ we found different lesional characteristics between men and women, and reporting of the adjusted rate for baseline clinical and lesional characteristics would be helpful. As already mentioned by Lansky et al, ${ }^{22}$ women tend to have increased inhospital mortality after both elective and primary PCI compared with men, but this difference becomes smaller after adjustment for women's older age, smaller body surface area, and comorbidities at presentation. We also found that this difference between men and women diminished after using propensity matching. In previous research, lack of use of a propensity score was often a source of bias with regard to baseline clinical and lesional characteristics appearing to be dissimilar between men and women. In our study, women had more clinical risk factors, but men had more specific lesions. Therefore, propensity score matching reflects the interplay between baseline clinical and lesional characteristics.

In our study, men had more extensive coronary lesions, so were likely to have longer stents and to undergo a greater number of stenting procedures. The increase in stent number 
and length is mainly attributable to increased target lesion revascularization and instent restenosis causing myocardial infarction and an increase in MACE. ${ }^{23,24}$ The outcome of unadjusted MACE with target vessel revascularization at two-year follow-up explains the discontinuation of dual antiplatelet therapy, and Brener et $\mathrm{al}^{25}$ suggested that prolonged dual antiplatelet therapy reduces MACE after PCI. A previous study by Harmsze et $\mathrm{al}^{26}$ concluded that many patients are resistant to the idea of anticoagulant medication, which can be a major cause of MACE after PCI. Like the work reported by Nguyen et al, ${ }^{27}$ our study contained more men than women, potentially reflecting a degree of gender bias when referring patients for coronary angiography. Certain clinical features, such as severity of disease, flow reserve, local inflammation, or lesion complexity might be surrogates for predicting outcomes. For example, the degree of intimal hyperplasia is significantly greater in lesions treated with longer stents. ${ }^{28}$

\section{Conclusion}

Although baseline clinical variables differed between women and men in this study, and women had less extensive coronary lesions than men, both genders had similar outcomes after drug-eluting stent implantation in this Chinese population. Our present study suffers from the limitations of any nonrandomized trial. The significant difference between the genders in terms of baseline demographics and the smaller number of women included could have led to bias in interpretation of the study results. Another limitation of this study was the use of medium-term clinical outcomes, which could account for the lack of a significant difference seen in outcomes after propensity score matching between men and women. Further, we did not investigate for differences in clinical outcome between the types of stent used. We also did not compare images obtained on intravascular ultrasound for men and women. Future research on the outcomes of drugeluting stent implantation should investigate intravascular ultrasound or fractional flow reserve in men and women, enabling translation of the results into real-world practice.

\section{Acknowledgments}

This work was funded by the Nanjing Municipal Health Outstanding Project (Grant 2000 NJMHOP-120). The study was also supported by a grant from the Science and Technology Bureau of Nanjing City (Grant 2009sc331029). The authors appreciate Professor Bao-Xiang Duan who headed the events committee. They also acknowledge the contributions of Kang Jin, Ling Lin, Hai-Mei Xu, Yin-Yin
Zhao, and Ling-Ling Liu in data collection and remote monitoring.

\section{Disclosure}

The authors report no conflicts of interest in this work.

\section{References}

1. Yeh RW, Charndra M, McCulloch CE, et al. Accounting for the mortality benefit of drug-eluting stents in percutaneous coronary intervention: a comparison of methods in a retrospective cohort study. BMC Med. 2011;9:78.

2. Ma J, Yang W, Singh M, et al. Meta-analysis of long-term outcomes of drug-eluting stent implantations for chronic total coronary occlusions. Heart Lung. 2011;40(3):e32-e40.

3. Woo M, Fan CQ, Chen YL, et al. Gender differences in patients undergoing coronary stenting in current stent era. Chin Med J (Engl). 2011; 124(6):862-866.

4. Braunwald E, Antman EM, Beasley JW, et al. ACC/AHA 2002 guidelines update for the management of patients with unstable angina and non-STsegment elevation myocardial infarction-summary article: a report of the American College of Cardiology/American Heart Association task force on practice guidelines (committee on the management of patients with unstable angina). J Am Coll Cardiol. 2002;40(7):1366-1374.

5. Antman EM, Anbe DT, Armstrong PW, et al. ACC/AHA guideline for the management of patient with ST-elevation myocardial infarction; a report of the American College of Cardiology/American Heart Association task force on practice guidelines (Committee to revise the 1999 guidelines for the management of patients with acute myocardial infarction). J Am Coll Cardiol. 2004;44(3):671-719.

6. Mauri L, Hsieh WH, Massaro JM, et al. Stent thrombosis in randomized clinical trials of drug-eluting stent. $N$ Engl J Med. 2007;356(10): 1020-1029.

7. Berger JS, Elliott L, Gallup D, et al. Sex differences in mortality following acute coronary syndromes. JAMA. 2009;302(8):874-882.

8. Jackson EA, Moscucci M, Smith D, et al. The association of sex with outcomes among patients undergoing primary percutaneous coronary intervention for ST elevation myocardial infarction in the contemporary era: insights from Blue Cross Blue Shield of Michigan Cardiovascular Consortium (BMC2). Am Heart J. 2011;161(1):106-112.

9. Williams JK, Adams MR, Klopfenstein HS. Estrogen modulates responses of atherosclerotic coronary arteries. Circulation. 1990;81(5): $1680-1687$.

10. Sabate M, Jimenez-Quevedo P, Angiolillo DJ, et al. Randomized comparison of sirolimus-eluting stent versus standard stent for percutaneous coronary revascularization in diabetic patients: the Diabetes and Sirolimus-Eluting Stent (DIABETES) trial. Circulation. 2005;112(14):2175-2183.

11. Niles NW, McGrath PD, Malenka D, et al. Survival of patients with diabetes and multivessel coronary artery disease after surgical or percutaneous coronary revascularization: result of a large regional prospective study. Northern New England Cardiovascular Disease Study Group. J Am Coll Cardiol. 2001;37(4):1008-1015.

12. Lincoff AM. Important triad in cardiovascular medicine: diabetes, coronary intervention, and platelet glycoprotein IIb/IIIa receptor blockade. Circulation. 2003;107(11):1556-1559.

13. Mehilli J, Kastrati A, Bollwein H, et al. Gender and restenosis after coronary artery stenting. Eur Heart J. 2003;24(16):1523-1530.

14. Mehilli J, Kastrati A, Dirschinger J, et al. Differences in prognostic factors and outcomes between women and men undergoing coronary artery stenting. JAMA. 2000;284(14):1799-1805.

15. Ogita M, Miyauchi K, Dohi T, et al. Gender-based outcomes among patients with diabetes mellitus after percutaneous coronary intervention in the drug-eluting stent era. Int Heart J. 2011;52(6): 348-352. 
16. Spinler SA, Mahaffey KW, Gallup D, et al. Relationship between renal function and outcomes in high-risk patients with non-ST-segment elevation acute coronary syndrome: result from SYNERGY. Int $J$ Cardiol. 2010;144(1):36-41.

17. Kralev S, Henning O, Lang S, et al. Sex-based differences in clinical and angiographic outcomes in patients with ST-elevation myocardial infarction treated with concomitant use of glycoprotein IIb/IIIa inhibitors. Cardiol J. 2010;17(6):580-586.

18. Lee KH, Jeong MH, Ahn YK, et al. Gender differences of success rate of percutaneous coronary intervention and short term cardiac events in Korea Acute Myocardial Infarction Registry. Int J Cardiol. 2008;130(2): 227-234.

19. Welty FK, Mittleman MA, Healy RW, et al. Similar results of percutaneous transluminal coronary angioplasty for women and men with post myocardial infarction ischemia. J Am Coll Cardiol. 1994; 23(1):35-39.

20. Schuhlen H, Kastrati A, Dirschinger J, et al. Intracoronary stenting and risk for major adverse cardiac events during the first month. Circulation. 1998;98(2):104-111.

21. Lansky AJ, Ng VG, Maehara A, et al. Gender and the extent of coronary atherosclerosis, plaque composition and clinical outcomes in the acute coronary syndrome. JACC Cardiovasc Imaging. 2012;5 Suppl 3: S62-S72.
22. Lansky AJ, Hochman JS, Ward PA, et al. Percutaneous coronary intervention and adjunctive pharmacotherapy in women. A statement for Healthcare Professionals from the American Heart Association. Circulation. 2005;111(7):940-953.

23. Zhang QY, Li Y, Guan SY, et al. Incidence and predictors of definite stent thrombosis after coronary stent implantation. Chin Med J (Engl). 2012;125(9):1547-1551.

24. Chang SH, Chen CC, Hsieh MJ, et al. Lesion length impacts long term outcomes of drug-eluting stent and bare metal stents differently. PLoS One. 2013;8(1):e53207.

25. Brener SJ, Steinhubl SR, Berger PB, et al. Prolonged dual antiplatelet therapy after percutaneous coronary intervention reduces ischemic events without affecting the need for repeat revascularization: insights from the CREDO trail. J Invasive Cardiol. 2007;19(7):287-290.

26. Harmsze AM, Van Werkum JW, Ten Berg JM, et al. CYP2C19*2 and CYP2C9*3 alleles are associated with stent thrombosis: a case-control study. Eur Heart J. 2010;(24):3046-3053.

27. Nguyen JT, Berger AK, Duval S, et al. Gender disparity in cardiac procedures and medication use for acute myocardial infarction. $\mathrm{Am}$ Heart J. 2008;155(5):862-868.

28. Kang SJ, Mintz GS, Park DW, et al. Mechanisms of in-stent restenosis after drug-eluting stent implantation: intravascular ultrasound analysis. Circ Cardiovasc Interv. 2011;4(1):9-14.
Patient Preference and Adherence

\section{Publish your work in this journal}

Patient Preference and Adherence is an international, peer-reviewed, open access journal focusing on the growing importance of patient preference and adherence throughout the therapeutic continuum. Patient satisfaction, acceptability, quality of life, compliance, persistence and their role in developing new therapeutic modalities and compounds to

\section{Dovepress}

optimize clinical outcomes for existing disease states are major areas of interest. This journal has been accepted for indexing on PubMed Central. The manuscript management system is completely online and includes a very quick and fair peer-review system. Visit http://www.dovepress.com/ testimonials.php to read real quotes from published authors. 